\title{
Homogeneous approximation property for continuous shearlet transforms in higher dimensions
}

\author{
Yu Su, ${ }^{1,2}$, Wanchang Zhang ${ }^{3 *}$ and Wenting $\mathrm{Su}^{2}$
}

\section{"Correspondence:}

zhangwc@radi.ac.cn

${ }^{3}$ Key Laboratory of Digital Earth

Science, Institute of Remote

Sensing and Digital Earth, Chinese

Academy of Sciences, No. 9

Dengzhuang South Road, Haidian

District, Beijing, 100094, China

Full list of author information is

available at the end of the article

\section{算 Springer}

\begin{abstract}
This paper is concerned with the generalization of the homogeneous approximation property (HAP) for a continuous shearlet transform to higher dimensions. First, we give a pointwise convergence result on the inverse shearlet transform in higher dimensions. Second, we show that every pair of admissible shearlets possess the HAP in the sense of $L^{2}\left(R^{d}\right)$. Third, we give a sufficient condition for the pointwise HAP to hold, which depends on both shearlets and functions to be reconstructed.
\end{abstract}

Keywords: homogeneous approximation property; continuous shearlet transform; shearlet; admissible

\section{Introduction}

Modern technology allows for easy creation, transmission, and storage of huge amounts of higher-dimensional data. Nowadays, the key problem is to extract the relevant information from these huge amounts of higher-dimensional data. In this context, one particular problem which is currently in the center of interest is the analysis of directional information in higher dimensions. Due to traditional wavelets' limited directional sensitivity, traditional wavelets are not very efficient in dealing with distributed discontinuities such as the edges occurring in natural images or the boundaries of solid bodies. Hence new direction representation systems have to be developed, such as ridgelets [1], curvelets [2], contourlets [3], shearlets [4, 5], and many others. Among all these approaches, the shearlet transform stands out because it is related to group theory, and it has a flexible enough extension to precisely detect the position and orientation of singularities and to provide optimally sparse representations.

In this paper, we study the homogeneous approximation property (HAP) for the shearlet transform in higher dimensions. The HAP is useful in practice, since it means that the approximation rate in a reconstruction of $f$ is invariant under time-scale shifts. The HAP was introduced by Ramanathan and Steger in [6]. Then it was studied for continuous wavelet systems and their discretization introduced by Sun [7-10], and for irregular wavelet frames introduced by Heil and Kutyniok in [11], and for Gabor systems in [12].

In the discrete shearlet system case, the HAP for irregular shearlet frames has been studied in $[13,14]$. Although the HAP is well understood for Gabor, wavelet, and discrete irregular shearlet frames, it is not very clear for continuous shearlet systems in higher

(c) 2016 Su et al. This article is distributed under the terms of the Creative Commons Attribution 4.0 International License (http://creativecommons.org/licenses/by/4.0/), which permits unrestricted use, distribution, and reproduction in any medium, provided you give appropriate credit to the original author(s) and the source, provide a link to the Creative Commons license, and indicate if changes were made. 
dimensions. Due to the HAP being particularly useful in both theoretical and numerical applications, and the urgent need for analyzing higher-dimensional data, there is an urgent need for studying the HAP for continuous shearlet systems in higher dimensions. This is exactly the concern of this paper.

The aim of this paper is threefold.

(i) We give a pointwise convergence result on the inverse shearlet transform in higher dimensions.

(ii) We show that every pair of admissible shearlets possess the HAP in the sense of $L^{2}\left(R^{d}\right)$.

(iii) We give a sufficient condition for the pointwise HAP to hold, which depends on both shearlets and functions to be reconstructed in higher dimensions.

This paper is organized as follows. In Section 2, we collect some basic notations and definitions. Then, in Section 3, we give a pointwise convergence result on the inverse shearlet transform in higher dimensions. In Section 4, we show that every pair of admissible shearlets possess the HAP in the sense of $L^{2}\left(R^{d}\right)$. Finally, in Section 5, we give a sufficient condition for the pointwise HAP to hold, which depends on both shearlets and functions to be reconstructed in higher dimensions.

\section{Notation}

The shearlet group $G=\left\{(a, s, t), a \in R^{+}, s \in R^{d-1}, t \in R^{d}\right\}$ equipped with group multiplication by

$$
(a, s, t)\left(a^{\prime}, s^{\prime}, t^{\prime}\right)=\left(a a^{\prime}, s+a^{\frac{d-1}{d}} s^{\prime}, t+S_{s} A_{a} t^{\prime}\right),
$$

where $s=\left(s_{1}, \ldots, s_{d-1}\right)^{T}$, and

$$
A_{a}=\left[\begin{array}{cc}
a & 0 \\
0 & a^{\frac{1}{d}} I_{d-1}
\end{array}\right], \quad S_{s}=\left[\begin{array}{cc}
1 & s^{T} \\
0 & I_{d-1}
\end{array}\right] .
$$

We have the mapping $\sigma: G \rightarrow \mathcal{U}\left(L^{2}\left(R^{d}\right)\right)$, where $\mathcal{U}\left(L^{2}\left(R^{d}\right)\right)$ denotes the group of unitary operators on $L^{2}\left(R^{d}\right)$, and it is a unitary representation of the shearlet group. The shearlet transform of $f \in L^{2}\left(R^{d}\right)$ with respect to $\psi \in L^{2}\left(R^{d}\right)$ is defined in the following way:

$$
S H_{\psi} f(a, s, t)=\left\langle f, \psi_{a, s, t}\right\rangle=\langle f, \sigma(a, s, t) \psi\rangle=\int_{R^{d}} f(x) \overline{\sigma(a, s, t) \psi(x)} d x,
$$

where

$$
\sigma(a, s, t) \psi(x)=a^{-\frac{2 d-1}{2 d}} \psi\left(A_{a}^{-1} S_{s}^{-1}(x-t)\right) .
$$

For $A_{2}>A_{1}>0$ and $B>0$, denote

$$
Q_{A_{1}, A_{2} ; B}=\left(\left[-A_{2},-A_{1}\right] \cup\left[A_{1}, A_{2}\right]\right) \times[-B, B]^{d-1} \times[-B, B]^{d}
$$

for every $(p, q, r) \in G$, its $\left(A_{1}, A_{2} ; B\right)$-neighborhood is defined by

$$
\begin{aligned}
(p, q, r) Q_{A_{1}, A_{2} ; B}= & \left\{(p, q, r)(a, s, t)=\left(p a, q+p^{\frac{d-1}{d}} s, r+S_{q} A_{p} t\right):\right. \\
& \left.a \in\left[-A_{2},-A_{1}\right] \cup\left[A_{1}, A_{2}\right], s \in[-B, B]^{d-1}, t \in[-B, B]^{d}\right\} .
\end{aligned}
$$


The Fourier transform of $f \in L^{1}\left(R^{d}\right) \cap L^{2}\left(R^{d}\right)$ is defined by

$$
\hat{f}(\omega)=\int_{R^{d}} f(x) e^{-2 \pi i\langle x, \omega\rangle} d x .
$$

In [15], we call a function $\psi \in L^{2}\left(R^{d}\right)$ admissible if

$$
C_{\psi}=\int_{R^{d}} \frac{|\hat{\psi}(\omega)|^{2}}{\left|\omega_{1}\right|^{d}} d \omega<\infty
$$

We need the condition of deformation of admissibility as follows:

$$
C_{\psi}=\int_{R^{d-1}} \int_{0}^{\infty}\left|\hat{\psi}\left(A_{a}^{T} S_{s}^{T} \omega\right)\right|^{2} \frac{d a d s}{a^{\frac{d^{2}-d+1}{d}}}<\infty
$$

it being independent of $\omega$ (up to a zero measured set) and $C_{\psi} \neq 0$.

In fact, by using a similar method to [16], we have

$$
\begin{aligned}
& \int_{R^{d-1}} \int_{R^{+}} \frac{\left|\hat{\psi}\left(A_{a}^{T} S_{s}^{T} \omega\right)\right|^{2}}{a^{\frac{d^{2}-d+1}{d}}} d a d s \\
& \quad=\int_{R} \ldots \int_{R} \int_{R^{+}} \frac{\left|\hat{\psi}\left(a \omega_{1}, a^{\frac{1}{d}}\left(s_{1} \omega_{1}+\omega_{2}\right), \ldots, a^{\frac{1}{d}}\left(s_{d-1} \omega_{1}+\omega_{d}\right)\right)^{T}\right|^{2}}{a^{\frac{d^{2}-d+1}{d}}} d a d s_{1} \cdots d s_{d-1} \\
& =\int_{R} \cdots \int_{R} \int_{R^{+}} \frac{\left|\hat{\psi}\left(a \omega_{1}, a^{\frac{1}{d}}\left(s_{1} \omega_{1}+\omega_{2}\right), \ldots, a^{\frac{1}{d}}\left(s_{d-2} \omega_{1}+\omega_{d-1}\right), v_{d}\right)^{T}\right|^{2}}{a^{\frac{d^{2}-d+1}{d}}} d a d s_{1} \cdots d s_{d-2} \frac{d v_{d-1}}{a^{\frac{1}{d}} \omega_{1}} \\
& =\int_{R} \ldots \int_{R} \int_{R} \frac{\left|\hat{\psi}\left(v_{1}, v_{2}, \ldots, v_{d}\right)^{T}\right|^{2}}{a^{\frac{d^{2}-d+1}{d}}} \frac{1}{a^{\frac{d-1}{d}} \omega_{1}^{d-1}} d a d v_{2} \cdots d v_{d} \\
& =\int_{R} \cdots \int_{R} \int_{R} \frac{\left|\hat{\psi}\left(v_{1}, v_{2}, \ldots, v_{d}\right)^{T}\right|^{2} \omega_{1}^{\frac{d^{2}-d+1}{d}}}{v_{1} \frac{d^{2}-d+1}{d}} \frac{\omega_{1}^{\frac{d-1}{d}}}{\omega_{1}^{d} v_{1}^{\frac{d-1}{d}}} d v_{1} d v_{2} \cdots d v_{d} \\
& =\int_{R^{d}} \frac{|\hat{\psi}(v)|^{2}}{v_{1}^{d}} d v=C_{\psi} \cdot
\end{aligned}
$$

In [17], we call a pair of functions $\left(\psi_{1}, \psi_{2}\right)$ admissible if both $\psi_{1}$ and $\psi_{2}$ are admissible and

$$
C_{\psi_{1}, \psi_{2}}=\int_{R^{d-1}} \int_{0}^{\infty} \overline{\hat{\psi}_{1}\left(A_{a}^{T} S_{s}^{T} \omega\right)} \hat{\psi}_{2}\left(A_{a}^{T} S_{s}^{T} \omega\right) \frac{d a d s}{a^{\frac{d^{2}-d+1}{d}}}<\infty
$$

is independent of $\omega$ (up to a zero measured set) and $C_{\psi_{1}, \psi_{2}} \neq 0$.

\section{Pointwise convergence of the inverse shearlet transforms}

In this section, we give a pointwise convergence result on the inverse shearlet transform. We show that $f \in L^{1}\left(R^{d}\right)$ and the admissibility of $\left(\psi_{1}, \psi_{2}\right)$ are enough to guarantee the pointwise convergence. We would like to mention that our ideas in this section are inspired by Liu and Sun [17].

We begin with a useful theorem. 
Theorem 3.1 Let $\psi_{1}, \psi_{2}$ be a pair of admissible shearlets with $C_{\psi_{1}, \psi_{2}} \neq 0$. For any $f \in$ $L^{2}\left(R^{d}\right)$ and $A_{2}>A_{1}>0$, define

$$
f_{A_{1}, A_{2}}(x)=C_{\psi_{1}, \psi_{2}}^{-1} \int_{A_{1}}^{A_{2}} d a \int_{R^{d-1}} d s \int_{R^{d}}\left\langle f, \sigma(a, s, t) \psi_{1}\right\rangle \sigma(a, s, t) \psi_{2}(x) \frac{d t}{a^{d+1}} .
$$

Then we have

$$
\hat{f}_{A_{1}, A_{2}}(\omega)=C_{\psi_{1}, \psi_{2}}^{-1} \hat{f}(\omega) \int_{R^{d-1}} \int_{A_{1}}^{A_{2}} \overline{\hat{\psi}_{1}\left(A_{a}^{T} S_{s}^{T} \omega\right)} \hat{\psi}_{2}\left(A_{a}^{T} S_{s}^{T} \omega\right) \frac{d a d s}{a^{\frac{d^{2}-d+1}{d}}} .
$$

Proof First, we show that $f_{A_{1}, A_{2}}$ is well defined on $R^{d}$. For any $x \in R^{d}$, we get

$$
\begin{aligned}
& \int_{A_{1}}^{A_{2}} \frac{d a}{a^{d+1}} \int_{R^{d-1}} d s \int_{R^{d}} \mid\left\langle f, \sigma(a, s, t) \psi_{1}\left|\sigma(a, s, t) \psi_{2}(x)\right| d t\right. \\
& \quad \leq \int_{A_{1}}^{A_{2}} \frac{d a}{a^{d+1}}\left(\int_{R^{d}} \int_{R^{d-1}}\left|\left\langle f, \sigma(a, s, t) \psi_{1}\right\rangle\right|^{2} d s d t\right)^{\frac{1}{2}}\left(\int_{R^{d}} \int_{R^{d-1}}\left|\sigma(a, s, t) \psi_{2}(x)\right|^{2} d s d t\right)^{\frac{1}{2}} \\
& \quad=\left(\int_{A_{1}}^{A_{2}} \frac{d a}{a^{d+1}}\right)^{\frac{1}{2}}\left(\int_{A_{1}}^{A_{2}} \frac{d a}{a^{d+1}} \int_{R^{d}} \int_{R}\left|\left\langle f, \sigma(a, s, t) \psi_{1}\right\rangle\right|^{2} d s d t\right)^{\frac{1}{2}}\left\|\psi_{2}(x)\right\|_{2} \\
& \quad=C_{\psi_{1}}^{\frac{1}{2}}\|f\|_{2}\left\|\psi_{2}(x)\right\|_{2}\left(\int_{A_{1}}^{A_{2}} \frac{d a}{a^{d+1}}\right)^{\frac{1}{2}} \\
& \quad=d^{-\frac{1}{2}} C_{\psi_{1}}^{\frac{1}{2}}\|f\|_{2}\left\|\psi_{2}(x)\right\|_{2}\left(A_{1}^{-d}-A_{2}^{-d}\right)^{\frac{1}{2}} \\
& <\infty
\end{aligned}
$$

Hence, $f_{A_{1}, A_{2}}$ is well defined on $R^{d}$.

Second, we show that $f_{A_{1}, A_{2}}$ is uniformly continuous on $R^{d}$. For any $x, x^{\prime} \in R^{d}$, we have

$$
\begin{aligned}
& \left|f_{A_{1}, A_{2}}(x)-f_{A_{1}, A_{2}}\left(x^{\prime}\right)\right| \\
& \left.=\left|C_{\psi_{1}, \psi_{2}}^{-1} \int_{A_{1}}^{A_{2}} d a \int_{R^{d-1}} d s \int_{R^{d}}\right| f, \sigma(a, s, t) \psi_{1}\right\rangle\left(\sigma(a, s, t) \psi_{2}(x)-\sigma(a, s, t) \psi_{2}\left(x^{\prime}\right)\right) \frac{d t}{a^{d+1}} \mid \\
& \leq \mid \\
& \quad C_{\psi_{1}, \psi_{2}}^{-1} \mid\left(\int_{R^{d}} \int_{R^{d-1}} \int_{A_{1}}^{A_{2}}|\hat{f}(\omega)|^{2}\left|\hat{\psi}_{1}\left(A_{a}^{T} S_{s}^{T} \omega\right)\right|^{2} \frac{d a d s d \omega}{\left.a^{\frac{d^{2}-d+1}{d}}\right)^{\frac{1}{2}}}\right. \\
& \quad \times\left(\int_{R^{d}} \int_{R^{d-1}} \int_{A_{1}}^{A_{2}} \mid a^{-\frac{2 d-1}{2 d}} \psi_{2}\left(A_{a}^{-1} S_{s}^{-1}(x-t)\right)\right. \\
& \left.\quad-\left.a^{-\frac{2 d-1}{2 d}} \psi_{2}\left(A_{a}^{-1} S_{s}^{-1}\left(x^{\prime}-t\right)\right)\right|^{2} \frac{d a d s d t}{a^{d+1}}\right)^{\frac{1}{2}} \\
& \leq\left|C_{\psi_{1}, \psi_{2}}^{-1}\right| C_{\psi_{1}}^{\frac{1}{2}} \mid \hat{f} \|_{2}\left(\int_{R^{d-1}} \int_{A_{1}}^{A_{2}}\left\|\psi_{2}\left(A_{a}^{-1} S_{s}^{-1} x-\cdot\right)-\psi_{2}\left(A_{a}^{-1} S_{s}^{-1} x^{\prime}-\cdot\right)\right\|_{2}^{2} \frac{d a d s}{a^{d+1}}\right)^{\frac{1}{2}} .
\end{aligned}
$$

Hence

$$
\lim _{\left\|x-x^{\prime}\right\|_{2} \rightarrow 0}\left|f_{A_{1}, A_{2}}(x)-f_{A_{1}, A_{2}}\left(x^{\prime}\right)\right|=0 .
$$

So, $f_{A_{1}, A_{2}}$ is uniformly continuous on $R^{d}$. 
Third, we prove (1). By (2), for any $g \in L^{1} \cap L^{2}\left(R^{d}\right)$,

$$
\int_{R^{d}}|g(x)| d x \int_{A_{1}}^{A_{2}} \frac{d a}{a^{d+1}} \int_{R^{d-1}} d s \int_{R^{d}} \mid\left\langle f, \sigma(a, s, t) \psi_{1}\left|\sigma(a, s, t) \psi_{2}(x)\right| d t<\infty .\right.
$$

We generalized the formula in [18],

$$
\begin{aligned}
\left\langle f, a^{-\frac{2 d-1}{2 d}} \psi\left(A_{a}^{-1} S_{s}^{-1}(x-t)\right)\right\rangle & =\langle f, \sigma(a, s, 0) \psi(x-t)\rangle \\
& =f * \sigma(a, s, 0) \psi^{*}(t) .
\end{aligned}
$$

By using (3), we have

$$
\begin{aligned}
\left\langle f_{A_{1}, A_{2}}, g\right\rangle & =C_{\psi_{1}, \psi_{2}}^{-1} \int_{A_{1}}^{A_{2}} d a \int_{R^{d-1}} d s \int_{R^{d}}\left\langle f, \sigma(a, s, t) \psi_{1}\right\rangle\left\langle\sigma(a, s, t) \psi_{2}(x), g\right\rangle \frac{d t}{a^{d+1}} \\
& =C_{\psi_{1}, \psi_{2}}^{-1} \int_{A_{1}}^{A_{2}} \frac{d a}{a^{d+1}} \int_{R^{d-1}} d s \int_{R^{d}} f * \sigma(a, s, 0) \psi_{1}^{*}(t) \overline{g * \sigma(a, s, 0) \psi_{2}^{*}(t)} d t \\
& =C_{\psi_{1}, \psi_{2}}^{-1} \int_{A_{1}}^{A_{2}} \frac{d a}{a^{d+1}} \int_{R^{d-1}} d s \int_{R^{d}} \hat{f}(\omega) \overline{\sigma(a, s, 0) \hat{\psi}_{1}(\omega) \sigma(a, s, 0) \hat{\psi}_{2}(\omega) \overline{\hat{g}(\omega)}} d \omega \\
& =C_{\psi_{1}, \psi_{2}}^{-1} \int_{R^{d}} \hat{f}(\omega) \overline{\hat{g}(\omega)} d \omega \int_{R^{d-1}} \int_{A_{1}}^{A_{2}} \overline{\sigma(a, s, 0) \hat{\psi}_{1}(\omega)} \sigma(a, s, 0) \hat{\psi}_{2}(\omega) \frac{d a d s}{a^{d+1}} \\
& =C_{\psi_{1}, \psi_{2}}^{-1} \int_{R^{d}} \hat{f}(\omega) \overline{\hat{g}(\omega)} d \omega \int_{R^{d-1}} \int_{A_{1}}^{A_{2}} \overline{\hat{\psi}_{1}\left(A_{a}^{T} S_{s}^{T} \omega\right)} \hat{\psi}_{2}\left(A_{a}^{T} S_{s}^{T} \omega\right) \frac{d a d s}{a^{\frac{d^{2}-d+1}{d}}} .
\end{aligned}
$$

Since $g \in L^{1} \cap L^{2}\left(R^{d}\right)$, we get

$$
\hat{f}_{A_{1}, A_{2}}(\omega)=C_{\psi_{1}, \psi_{2}}^{-1} \hat{f}(\omega) \int_{R^{d-1}} \int_{A_{1}}^{A_{2}} \overline{\hat{\psi}_{1}\left(A_{a}^{T} S_{s}^{T} \omega\right)} \hat{\psi}_{2}\left(A_{a}^{T} S_{s}^{T} \omega\right) \frac{d a d s}{a^{d^{2}-d+1}}
$$

We can see that when $d=2$, this result is suitable for the two dimensional case, so our result covers the bidimension case.

Next we give a convergence result on the inverse shearlet transform. Because only the admissibility is invoked, the following theorem improves Theorem 3.1.

Theorem 3.2 Let $\left(\psi_{1}, \psi_{2}\right)$ be a pair of admissible shearlets, and $C_{\psi_{1}, \psi_{2}} \neq 0$.

(i) For any $f \in L^{2}\left(R^{d}\right)$, we have

$$
\lim _{\substack{A_{1} \rightarrow 0 \\ A_{2} \rightarrow \infty}}\left\|f-f_{A_{1}, A_{2}}\right\|_{2}=0
$$

(ii) For any $\hat{f} \in L^{1}\left(R^{d}\right)$, we have

$$
\lim _{\substack{A_{1} \rightarrow 0 \\ A_{2} \rightarrow \infty}}\left\|f-f_{A_{1}, A_{2}}\right\|_{\infty}=0
$$

Proof We only prove the second part, the first part can be proved similarly. 
By using Theorem 3.1, we have

$$
\begin{aligned}
\left\|f-f_{A_{1}, A_{2}}\right\|_{\infty} & \leq\left\|\hat{f}-\hat{f}_{A_{1}, A_{2}}\right\|_{1} \\
& =\int_{R^{d}}|\hat{f}(\omega)| \cdot\left|1-C_{\psi_{1}, \psi_{2}}^{-1} \int_{R^{d-1}} \int_{A_{1}}^{A_{2}} \overline{\hat{\psi}_{1}\left(A_{a}^{T} S_{s}^{T} \omega\right)} \hat{\psi}_{2}\left(A_{a}^{T} S_{s}^{T} \omega\right) \frac{d a d s}{a^{\frac{d^{2}-d+1}{d}}}\right| d \omega .
\end{aligned}
$$

Because both $\psi_{1}$ and $\psi_{2}$ are admissible, we have

$$
\begin{aligned}
& \left|\int_{R^{d-1}} \int_{A_{1}}^{A_{2}} \overline{\hat{\psi}_{1}\left(A_{a}^{T} S_{s}^{T} \omega\right)} \hat{\psi}_{2}\left(A_{a}^{T} S_{s}^{T} \omega\right) \frac{d a d s}{a^{\frac{d^{2}-d+1}{d}}}\right| \\
& \quad \leq\left|\int_{R^{d-1}} \int_{0}^{\infty} \overline{\hat{\psi}_{1}\left(A_{a}^{T} S_{s}^{T} \omega\right)} \hat{\psi}_{2}\left(A_{a}^{T} S_{s}^{T} \omega\right) \frac{d a d s}{a^{\frac{d^{2}-d+1}{d}}}\right| \\
& \quad \leq\left(\int_{R^{d-1}} \int_{0}^{\infty}\left|\hat{\psi}_{1}\left(A_{a}^{T} S_{s}^{T} \omega\right)\right|^{2} \frac{d a d s}{a^{\frac{d^{2}-d+1}{d}}}\right)^{\frac{1}{2}}\left(\int_{R^{d-1}} \int_{0}^{\infty}\left|\hat{\psi}_{2}\left(A_{a}^{T} S_{s}^{T} \omega\right)\right|^{2} \frac{d a d s}{a^{\frac{d^{2}-d+1}{d}}}\right)^{\frac{1}{2}} \\
& \quad=C_{\psi_{1}}^{\frac{1}{2}} C_{\psi_{2}}^{\frac{1}{2}}<\infty
\end{aligned}
$$

and

$$
\lim _{\substack{A_{1} \rightarrow 0 \\ A_{2} \rightarrow \infty}}\left|1-C_{\psi_{1}, \psi_{2}}^{-1} \int_{R^{d-1}} \int_{A_{1}}^{A_{2}} \overline{\hat{\psi}_{1}\left(A_{a}^{T} S_{s}^{T} \omega\right)} \hat{\psi}_{2}\left(A_{a}^{T} S_{s}^{T} \omega\right) \frac{d a d s}{a^{\frac{d^{2}-d+1}{d}}}\right|=0 .
$$

By using the dominated convergence theorem, we have the conclusion.

We can see that when $d=2$, this result is suitable for the two dimensional case, so our result covers the bidimension case.

\section{Homogeneous approximation property for continuous shearlet transforms in $L^{2}\left(R^{d}\right)$}

In this section, we study the HAP for continuous shearlet transforms in $L^{2}\left(R^{d}\right)$.

Theorem 4.1 If $\psi_{1}, \psi_{2} \in L^{2}\left(R^{d}\right)$ are a pair of admissible shearlets and $C_{\psi_{1}, \psi_{2}} \neq 0, f \in$ $L^{2}\left(R^{d}\right)$, and $\hat{f} \in L^{1}\left(R^{d}\right)$, then, for any $\varepsilon>0$, there exist some $A_{2}>A_{1}>0$, such that, for any $(p, q, r) \in G$ with any $0<A_{1}^{\prime} \leq A_{1}$ and $A_{2} \leq A_{2}^{\prime}$, we have

$$
\begin{aligned}
& \| \sigma(p, q, r) f-C_{\psi_{1}, \psi_{2}}^{-1} \iiint_{(a, s, t) \in(p, q, r) Q_{A_{1}^{\prime}, A_{2}^{\prime} ; B^{\prime}}}\left\langle\sigma(p, q, r) f, \sigma(a, s, t) \psi_{1}\right\rangle \\
& \quad \times \sigma(a, s, t) \psi_{2} \frac{d a d s d t}{a^{d+1}} \|_{2}^{2}<\varepsilon .
\end{aligned}
$$

Proof In fact, we have

$$
\begin{aligned}
& \| \sigma(p, q, r) f-C_{\psi_{1}, \psi_{2}}^{-1} \iiint_{(a, s, t) \in(p, q, r) Q_{A_{1}^{\prime}, A_{2}^{\prime} ; B^{\prime}}}\left\langle\sigma(p, q, r) f, \sigma(a, s, t) \psi_{1}\right\rangle \\
& \quad \times \sigma(a, s, t) \psi_{2} \frac{d a d s d t}{a^{d+1}} \|_{2}^{2}
\end{aligned}
$$




$$
\begin{aligned}
= & \sup _{\|g\|=1} \mid\left\langle\sigma(p, q, r) f-C_{\psi_{1}, \psi_{2}}^{-1} \iiint_{(a, s, t) \in(p, q, r) Q_{A_{1}^{\prime}, A_{2}^{\prime}: B^{\prime}}}\left\langle\sigma(p, q, r) f, \sigma(a, s, t) \psi_{1}\right\rangle\right. \\
& \left.\times \sigma(a, s, t) \psi_{2} \frac{d a d s d t}{a^{d+1}}, g\right\rangle\left.\right|^{2} \\
= & \sup _{\|g\|=1}\left|C_{\psi_{1}, \psi_{2}}^{-1} \iiint_{(a, s, t) \notin(p, q, r) Q_{A_{1}^{\prime}, A_{2}^{\prime} ; B^{\prime}}}\left\langle\sigma(p, q, r) f, \sigma(a, s, t) \psi_{1}\right\rangle\left\langle\sigma(a, s, t) \psi_{2}, g\right\rangle \frac{d a d s d t}{a^{d+1}}\right|^{2} \\
\leq & \left|C_{\psi_{1}, \psi_{2}}^{-2}\right| \iiint_{(a, s, t) \notin(p, q, r) Q_{A_{1}^{\prime}, A_{2}^{\prime} ; B^{\prime}}}\left|\left\langle\sigma(p, q, r) f, \sigma(a, s, t) \psi_{1}\right\rangle\right|^{2} \frac{d a d s d t}{a^{d+1}} \\
& \times \sup _{\|g\|=1} \iiint_{G}\left|\left\langle g, \sigma(a, s, t) \psi_{2}\right\rangle\right|^{2} \frac{d a d s d t}{a^{d+1}} \\
= & C_{\psi_{2}}\left|C_{\psi_{1}, \psi_{2}}^{-2}\right| \iiint_{(a, s, t) \notin(p, q, r) Q_{A_{1}^{\prime}, A_{2}^{\prime} ; B^{\prime}}}\left|\left\langle\sigma(p, q, r) f, \sigma(a, s, t) \psi_{1}\right\rangle\right|^{2} \frac{d a d s d t}{a^{d+1}} \\
= & C_{\psi_{2}}\left|C_{\psi_{1}, \psi_{2}}^{-2}\right| \iiint_{(a, s, t) \notin Q_{A_{1}^{\prime}, A_{2}^{\prime} ; B^{\prime}}}\left|\left\langle f, \sigma(a, s, t) \psi_{1}\right\rangle\right|^{2} \frac{d a d s d t}{a^{d+1}} \\
\leq & C_{\psi_{2}}\left|C_{\psi_{1}, \psi_{2}}^{-2}\right| \iiint_{(a, s, t) \notin Q_{A_{1}, A_{2} ; B}}\left|\left\langle f, \sigma(a, s, t) \psi_{1}\right\rangle\right|^{2} \frac{d a d s d t}{a^{d+1}} \\
:= & E_{A_{1}, A_{2} ; B}
\end{aligned}
$$

We can make $E_{A_{1}, A_{2} ; B}$ arbitrarily small by choosing $A_{1}$ small enough and $A_{2}$ and $B$ large enough. This completes the proof.

We can see that when $d=2$, this result is suitable for the two dimensional case, so our result covers the bidimension case.

\section{Homogeneous approximation property for continuous shearlet transforms in $L^{\infty}\left(R^{d}\right)$}

In this section, we study the HAP for continuous shearlet transforms in $L^{\infty}\left(R^{d}\right)$. We show that the pointwise HAP depends on both the shearlets and the functions to be reconstructed, which is quite different from the case of $L^{2}\left(R^{d}\right)$.

Theorem 5.1 is very different from the univariate continuous wavelet transform case [7], it is only a sufficient but not necessary condition for the pointwise HAP for a continuous shearlet transform to hold in $L^{\infty}\left(R^{d}\right)$.

Theorem 5.1 If $\psi_{1}, \psi_{2} \in L^{2}\left(R^{d}\right)$ are a pair of admissible shearlets and $C_{\psi_{1}, \psi_{2}} \neq 0, f \in$ $L^{2}\left(R^{d}\right)$, and $\hat{f} \in L^{1}\left(R^{d}\right)$, then, for any $\varepsilon, p_{0}>0$, there exist some $A_{2}>A_{1}>0$ such that, for any $(p, q, r) \in G$ with $0<p_{0} \leq p$ and any $0<A_{1}^{\prime} \leq A_{1}$ and $A_{2} \leq A_{2}^{\prime}$, we have

$$
\begin{aligned}
& \| \sigma(p, q, r) f(x)-C_{\psi_{1}, \psi_{2}}^{-1} \int_{A_{1}^{\prime} p}^{A_{2}^{\prime} p} d a \int_{R^{d-1}} d s \int_{R^{d}}\left\langle\sigma(p, q, r) f, \sigma(a, s, t) \psi_{1}\right\rangle \\
& \quad \times \sigma(a, s, t) \psi_{2}(x) \frac{d t}{a^{d+1}} \|_{\infty} \leq \varepsilon .
\end{aligned}
$$


Proof By using Theorem 3.1, we have

$$
\begin{aligned}
& \| \sigma(p, q, r) f(x)-C_{\psi_{1}, \psi_{2}}^{-1} \int_{A_{1}^{\prime} p}^{A_{2}^{\prime} p} d a \int_{R^{d-1}} d s \int_{R^{d}}\left\langle\sigma(p, q, r) f, \sigma(a, s, t) \psi_{1}\right\rangle \\
& \times \sigma(a, s, t) \psi_{2}(x) \frac{d t}{a^{d+1}} \|_{\infty} \\
& \leq\left|C_{\psi_{1}, \psi_{2}}^{-1}\right|\left|\int_{0}^{A_{1}^{\prime} p} d a \int_{R^{d-1}} d s \int_{R^{d}}\left\langle\sigma(p, q, r) f, \sigma(a, s, t) \psi_{1}\right\rangle \sigma(a, s, t) \psi_{2}(x) \frac{d t}{a^{d+1}}\right| \\
& +\left|C_{\psi_{1}, \psi_{2}}^{-1}\right|\left|\int_{A_{2}^{\prime} p}^{+\infty} d a \int_{R^{d-1}} d s \int_{R^{d}}\left\langle\sigma(p, q, r) f, \sigma(a, s, t) \psi_{1}\right\rangle \sigma(a, s, t) \psi_{2}(x) \frac{d t}{a^{d+1}}\right| \\
& \leq\left|C_{\psi_{1}, \psi_{2}}^{-1}\right| \int_{R^{d}}\left|p^{\frac{2 d-1}{2 d}} f\left(A_{p}^{T} S_{q}^{T} \omega\right)\right|\left|\int_{R^{d-1}} \int_{0}^{A_{1}^{\prime} p} \overline{\psi_{1}\left(A_{a}^{T} S_{s}^{T} \omega\right)} \psi_{2}\left(A_{a}^{T} S_{s}^{T} \omega\right) \frac{d a d s}{a^{\frac{d^{2}-d+1}{d}}}\right| d \omega \\
& +\left|C_{\psi_{1}, \psi_{2}}^{-1}\right| \int_{R^{d}}\left|p^{\frac{2 d-1}{2 d}} f\left(A_{p}^{T} S_{q}^{T} \omega\right)\right|\left|\int_{R^{d-1}} \int_{A_{2}^{\prime} p}^{+\infty} \overline{\psi_{1}\left(A_{a}^{T} S_{s}^{T} \omega\right)} \psi_{2}\left(A_{a}^{T} S_{s}^{T} \omega\right) \frac{d a d s}{a^{\frac{d^{2}-d+1}{d}}}\right| d \omega \\
& \leq\left|C_{\psi_{1}, \psi_{2}}^{-1}\right|\left|p_{0}\right|^{-\frac{2 d-1}{2 d}} \int_{R^{d}}|f(\omega)|\left(\int_{R^{d-1}} \int_{0}^{A_{1}^{\prime}}\left|\overline{\psi_{1}\left(A_{a}^{T} S_{s}^{T} \omega\right)} \psi_{2}\left(A_{a}^{T} S_{s}^{T} \omega\right)\right|^{2} \frac{d a d s}{a^{\frac{d^{2}-d+1}{d}}}\right)^{\frac{1}{2}} d \omega \\
& +\left|C_{\psi_{1}, \psi_{2}}^{-1}\right|\left|p_{0}\right|^{-\frac{2 d-1}{2 d}} \int_{R^{d}}|f(\omega)| \\
& \times\left(\int_{R^{d-1}} \int_{A_{2}^{\prime}}^{+\infty}\left|\overline{\psi_{1}\left(A_{a}^{T} S_{s}^{T} \omega\right)} \psi_{2}\left(A_{a}^{T} S_{s}^{T} \omega\right)\right|^{2} \frac{d a d s}{a^{\frac{d^{2}-d+1}{d}}}\right)^{\frac{1}{2}} d \omega \\
& =\mathrm{I}+\mathrm{II} \text {. }
\end{aligned}
$$

Because both $\psi_{1}$ and $\psi_{2}$ are admissible, we have

$$
\left(\int_{R^{d-1}} \int_{0}^{A_{1}^{\prime}}\left|\overline{\psi_{1}\left(A_{a}^{T} S_{s}^{T} \omega\right)} \psi_{2}\left(A_{a}^{T} S_{s}^{T} \omega\right)\right|^{2} \frac{d a d s}{a^{\frac{d^{2}-d+1}{d}}}\right)^{\frac{1}{2}} \leq C_{\psi_{1}}^{\frac{1}{2}} C_{\psi_{2}}^{\frac{1}{2}}<\infty
$$

and

$$
\left(\left.\int_{R^{d-1}} \int_{A_{2}^{\prime}}^{+\infty} \overline{\psi_{1}\left(A_{a}^{T} S_{s}^{T} \omega\right)} \psi_{2}\left(A_{a}^{T} S_{s}^{T} \omega\right)\right|^{2} \frac{d a d s}{a^{\frac{d^{2}-d+1}{d}}}\right)^{\frac{1}{2}} \leq C_{\psi_{1}}^{\frac{1}{2}} C_{\psi_{2}}^{\frac{1}{2}}<\infty
$$

First, for (4), we have

$$
\lim _{A_{1}^{\prime} \rightarrow 0}\left(\left.\int_{R^{d-1}} \int_{0}^{A_{1}^{\prime}} \overline{\mid \psi_{1}\left(A_{a}^{T} S_{s}^{T} \omega\right)} \psi_{2}\left(A_{a}^{T} S_{s}^{T} \omega\right)\right|^{2} \frac{d a d s}{a^{\frac{d^{2}-d+1}{d}}}\right)^{\frac{1}{2}}=0 .
$$

Second, for (5),

$$
\begin{aligned}
& \left.\int_{R^{d-1}} \int_{0}^{A_{2}^{\prime}} \overline{\mid \psi_{1}\left(A_{a}^{T} S_{s}^{T} \omega\right)} \psi_{2}\left(A_{a}^{T} S_{s}^{T} \omega\right)\right|^{2} \frac{d a d s}{a^{\frac{d^{2}-d+1}{d}}} \\
& \quad+\int_{R^{d-1}} \int_{A_{2}^{\prime}}^{+\infty}\left|\overline{\psi_{1}\left(A_{a}^{T} S_{s}^{T} \omega\right)} \psi_{2}\left(A_{a}^{T} S_{s}^{T} \omega\right)\right|^{2} \frac{d a d s}{a^{\frac{d^{2}-d+1}{d}}}
\end{aligned}
$$




$$
\begin{aligned}
& =\int_{R^{d-1}} \int_{0}^{\infty}\left|\overline{\psi_{1}\left(A_{a}^{T} S_{s}^{T} \omega\right)} \psi_{2}\left(A_{a}^{T} S_{s}^{T} \omega\right)\right|^{2} \frac{d a d s}{a^{\frac{d^{2}-d+1}{d}}} \\
& \leq C_{\psi_{1}} C_{\psi_{2}}<\infty .
\end{aligned}
$$

Hence, for any $\varepsilon_{1}>0$, there exists $M>0$, such that, for $A_{2}^{\prime}>M$, we have

$$
\begin{aligned}
& \left|\int_{R^{d-1}} \int_{0}^{A_{2}^{\prime}} \overline{\mid \psi_{1}\left(A_{a}^{T} S_{s}^{T} \omega\right)} \psi_{2}\left(A_{a}^{T} S_{s}^{T} \omega\right)\right|^{2} \frac{d a d s}{a^{\frac{d^{2}-d+1}{d}}} \\
& \quad-\left.\int_{R^{d-1}} \int_{0}^{\infty} \overline{\mid \psi_{1}\left(A_{a}^{T} S_{s}^{T} \omega\right)} \psi_{2}\left(A_{a}^{T} S_{s}^{T} \omega\right)\right|^{2} \frac{d a d s}{a^{\frac{d^{2}-d+1}{d}}} \mid<\varepsilon_{1}
\end{aligned}
$$

so we get

$$
\begin{gathered}
\left.\lim _{A_{2}^{\prime} \rightarrow \infty} \int_{R^{d-1}} \int_{0}^{A_{2}^{\prime}} \overline{\mid \psi_{1}\left(A_{a}^{T} S_{s}^{T} \omega\right)} \psi_{2}\left(A_{a}^{T} S_{s}^{T} \omega\right)\right|^{2} \frac{d a d s}{a^{\frac{d^{2}-d+1}{d}}} \\
\quad=\int_{R^{d-1}} \int_{0}^{\infty}\left|\overline{\psi_{1}\left(A_{a}^{T} S_{s}^{T} \omega\right)} \psi_{2}\left(A_{a}^{T} S_{s}^{T} \omega\right)\right|^{2} \frac{d a d s}{a^{\frac{d^{2}-d+1}{d}}} .
\end{gathered}
$$

Putting (7) in (6), we have

$$
\lim _{A_{2}^{\prime} \rightarrow+\infty}\left(\int_{R^{d-1}} \int_{A_{2}^{\prime}}^{+\infty}\left|\overline{\psi_{1}\left(A_{a}^{T} S_{s}^{T} \omega\right)} \psi_{2}\left(A_{a}^{T} S_{s}^{T} \omega\right)\right|^{2} \frac{d a d s}{a^{\frac{d^{2}-d+1}{d}}}\right)^{\frac{1}{2}}=0 .
$$

By the dominated convergence theorem, we have

$$
\begin{aligned}
& \lim _{A_{1}^{\prime} \rightarrow 0} \int_{R^{d}}|f(\omega)|\left(\int_{R^{d-1}} \int_{0}^{A_{1}^{\prime}}\left|\overline{\psi_{1}\left(A_{a}^{T} S_{s}^{T} \omega\right)} \psi_{2}\left(A_{a}^{T} S_{s}^{T} \omega\right)\right|^{2} \frac{d a d s}{a^{\frac{d^{2}-d+1}{d}}}\right)^{\frac{1}{2}} d \omega=0, \\
& \lim _{A_{2}^{\prime} \rightarrow \infty} \int_{R^{d}}|f(\omega)|\left(\left.\int_{R^{d-1}} \int_{A_{2}^{\prime}}^{+\infty} \overline{\mid \psi_{1}\left(A_{a}^{T} S_{s}^{T} \omega\right)} \psi_{2}\left(A_{a}^{T} S_{s}^{T} \omega\right)\right|^{2} \frac{d a d s}{a^{\frac{d^{2}-d+1}{d}}}\right)^{\frac{1}{2}} d \omega=0 .
\end{aligned}
$$

Hence, we can choose some $0<A_{1} \leq A_{2}$ such that, for any $0<p_{0} \leq p$, and any $0<A_{1}^{\prime} \leq A_{1}$ and $A_{2} \leq A_{2}^{\prime}$, we have

$$
\begin{aligned}
& \mathrm{I} \leq \frac{\varepsilon}{2}, \\
& \mathrm{II} \leq \frac{\varepsilon}{2} .
\end{aligned}
$$

Putting (8) and (9) together, we get the conclusion.

Theorem 5.2 If $\psi_{1}, \psi_{2} \in L^{2}\left(R^{d}\right)$ are a pair of admissible shearlets and $C_{\psi_{1}, \psi_{2}} \neq 0, f \in$ $L^{2}\left(R^{d}\right)$, and $\hat{f} \in L^{1}\left(R^{d}\right)$, then the following assertions are equivalent.

(i) There is some $x_{0} \in R^{d}$ such that, for any $\varepsilon>0$, there exist $0<A_{1}<A_{2}$ and any $(p, q, r) \in G, A_{2} \leq A_{2}^{\prime}, 0<A_{1}^{\prime} \leq A_{1}$, such that

$$
\begin{aligned}
& \mid \sigma(p, q, r) f\left(x_{0}\right)-C_{\psi_{1}, \psi_{2}}^{-1} \int_{A_{1}^{\prime} p}^{A_{2}^{\prime} p} d a \int_{R^{d-1}} d s \int_{R^{d}}\left\langle\sigma(p, q, r) f, \sigma(a, s, t) \psi_{1}\right\rangle \\
& \times \sigma(a, s, t) \psi_{2}\left(x_{0}\right) \frac{d t}{a^{d+1}} \mid \leq \varepsilon .
\end{aligned}
$$


(ii) For any $\varepsilon>0$ and $x \in R^{d}$, there exist $0<A_{1}<A_{2}$ and any $(p, q, r) \in G, A_{2} \leq A_{2}^{\prime}$,

$0<A_{1}^{\prime} \leq A_{1}$, such that

$$
\begin{aligned}
& \mid \sigma(p, q, r) f(x)-C_{\psi_{1}, \psi_{2}}^{-1} \int_{A_{1}^{\prime} p}^{A_{2}^{\prime} p} d a \int_{R^{d-1}} d s \int_{R^{d}}\left\langle\sigma(p, q, r) f, \sigma(a, s, t) \psi_{1}\right\rangle \\
& \quad \times \sigma(a, s, t) \psi_{2}(x) \frac{d t}{a^{d+1}} \mid \leq \varepsilon .
\end{aligned}
$$

(iii) There exist $0<A_{1}<A_{2}$ and any $x \in R^{d}, A_{2} \leq A_{2}^{\prime}, 0<A_{1}^{\prime} \leq A_{1}$, such that

$$
f(x)=C_{\psi_{1}, \psi_{2}}^{-1} \int_{A_{1}^{\prime}}^{A_{2}^{\prime}} d a \int_{R^{d-1}} d s \int_{R^{d}}\left(f, \sigma(a, s, t) \psi_{1}\right) \sigma(a, s, t) \psi_{2}(x) \frac{d t}{a^{d+1}}
$$

(iv) There exist $0<A_{1}<A_{2}, A_{2} \leq A_{2}^{\prime}$ and $0<A_{1}^{\prime} \leq A_{1}$, such that

$$
C_{\psi_{1}, \psi_{2}}=\int_{R^{d-1}} \int_{A_{1}^{\prime}}^{A_{2}^{\prime}} \overline{\hat{\psi}_{1}\left(A_{a}^{T} S_{s}^{T} \omega\right)} \hat{\psi}_{2}\left(A_{a}^{T} S_{s}^{T} \omega\right) \frac{d a d s}{a^{\frac{d^{2}-d+1}{d}}}
$$

(v) There exist $0<A_{1}<A_{2}$ and any $(p, q, r) \in G, A_{2} \leq A_{2}^{\prime}, 0<A_{1}^{\prime} \leq A_{1}$, such that

$$
\begin{aligned}
& \sigma(p, q, r) f(x) \\
& =C_{\psi_{1}, \psi_{2}}^{-1} \int_{A_{1}^{\prime} p}^{A_{2}^{\prime} p} d a \int_{R^{d-1}} d s \int_{R^{d}}\left\langle\sigma(p, q, r) f, \sigma(a, s, t) \psi_{1}\right\rangle \\
& \quad \times \sigma(a, s, t) \psi_{2}(x) \frac{d t}{a^{d+1}} .
\end{aligned}
$$

Proof For any $0<A_{1}<A_{2}$, we have

$$
f_{A_{1}, A_{2}}(x)=C_{\psi_{1}, \psi_{2}}^{-1} \int_{A_{1}}^{A_{2}} d a \int_{R^{d-1}} d s \int_{R^{d}}\left\langle f, \sigma(a, s, t) \psi_{1}\right\rangle \sigma(a, s, t) \psi_{2}(x) \frac{d t}{a^{d+1}}
$$

Then, for any $(p, q, r) \in G$, we have

$$
\begin{aligned}
& \sigma(p, q, r) f_{A_{1}, A_{2}}(x) \\
& \quad=C_{\psi_{1}, \psi_{2}}^{-1} \int_{A_{1} p}^{A_{2} p} d a \int_{R^{d-1}} d s \int_{R^{d}}\left\langle\sigma(p, q, r) f, \sigma(a, s, t) \psi_{1}\right\rangle \sigma(a, s, t) \psi_{2}(x) \frac{d t}{a^{d+1}} .
\end{aligned}
$$

(i) $\Rightarrow$ (ii): Assume that (i) holds, by substituting $r+x_{0}-x$ for $r$ in (10), we get

$$
\begin{aligned}
& \mid \sigma(p, q, r) f(x)-C_{\psi_{1}, \psi_{2}}^{-1} \int_{A_{1}^{\prime} p}^{A_{2}^{\prime} p} d a \int_{R^{d-1}} d s \int_{R^{d}}\left\langle\sigma\left(p, q, r+x_{0}-x\right) f, \sigma(a, s, t) \psi_{1}\right\rangle \\
& \quad \times \sigma(a, s, t) \psi_{2}\left(x_{0}\right) \frac{d t}{a^{d+1}} \mid \leq \varepsilon .
\end{aligned}
$$


Sue et al. Journal of Inequalities and Applications ( 2016) 2016:177

Page 11 of 13

On the other hand, by a change of variable of the form $t \rightarrow t+x_{0}-x$, we get

$$
\begin{gathered}
\int_{A_{1}^{\prime} p}^{A_{2}^{\prime} p} d a \int_{R^{d-1}} d s \int_{R^{d}}\left\langle\sigma\left(p, q, r+x_{0}-x\right) f, \sigma(a, s, t) \psi_{1}\right\rangle \sigma(a, s, t) \psi_{2}\left(x_{0}\right) \frac{d t}{a^{d+1}} \\
=\int_{A_{1}^{\prime} p}^{A_{2}^{\prime} p} d a \int_{R^{d-1}} d s \int_{R^{d}}\left\langle\sigma(p, q, r) f, \sigma(a, s, t) \psi_{1}\right\rangle \sigma(a, s, t) \psi_{2}(x) \frac{d t}{a^{d+1}} .
\end{gathered}
$$

Hence (ii) holds.

(ii) $\Rightarrow$ (iii):

$$
\begin{aligned}
& \left|f(x)-f_{A_{1}^{\prime}, A_{1}^{\prime}}(x)\right| \\
& =|p|^{\frac{2 d-1}{2 d}}\left|\sigma(p, q, r) f(x)-\sigma(p, q, r) f_{A_{1}^{\prime}, A_{1}^{\prime}}(x)\right| \\
& =|p|^{\frac{2 d-1}{2 d}} \mid \sigma(p, q, r) f(x)-C_{\psi_{1}, \psi_{2}}^{-1} \int_{A_{1}^{\prime} p}^{A_{2}^{\prime} p} d a \int_{R^{d-1}} d s \int_{R^{d}}\left\langle\sigma(p, q, r) f, \sigma(a, s, t) \psi_{1}\right\rangle \\
& \quad \times \sigma(a, s, t) \psi_{2}(x) \frac{d t}{a^{d+1}} \mid \\
& <|p|^{\frac{2 d-1}{2 d}} \varepsilon .
\end{aligned}
$$

Since $p$ is arbitrary, for any $x \in R^{d}, A_{2} \leq A_{2}^{\prime}, 0<A_{1}^{\prime} \leq A_{1}$, such that

$$
\begin{aligned}
f(x) & =f_{A_{1}^{\prime}, A_{1}^{\prime}}(x) \\
& =C_{\psi_{1}, \psi_{2}}^{-1} \int_{A_{1}^{\prime}}^{A_{2}^{\prime}} d a \int_{R^{d-1}} d s \int_{R^{d}}\left\langle f, \sigma(a, s, t) \psi_{1}\right\rangle \sigma(a, s, t) \psi_{2}(x) \frac{d t}{a^{d+1}} .
\end{aligned}
$$

(iii) $\Rightarrow$ (iv): For any $0<A_{1}^{\prime} \leq A_{1}$ and $A_{2} \leq A_{2}^{\prime}$

$$
\begin{aligned}
& \left|C_{\psi_{1}, \psi_{2}}-\int_{R^{d-1}} \int_{A_{1}^{\prime}}^{A_{2}^{\prime}} \overline{\hat{\psi}_{1}\left(A_{a}^{T} S_{s}^{T} \omega\right)} \hat{\psi}_{2}\left(A_{a}^{T} S_{s}^{T} \omega\right) \frac{d a d s}{a^{\frac{d^{2}-d+1}{d}}}\right| \\
& \quad \leq\left|C_{\psi_{1}, \psi_{2}}\right|+\int_{R^{d-1}} \int_{A_{1}^{\prime}}^{A_{2}^{\prime}} \overline{\mid \hat{\psi}_{1}\left(A_{a}^{T} S_{s}^{T} \omega\right)} \hat{\psi}_{2}\left(A_{a}^{T} S_{s}^{T} \omega\right) \mid \frac{d a d s}{a^{\frac{d^{2}-d+1}{d}}} \\
& \quad \leq\left|C_{\psi_{1}, \psi_{2}}\right|+C_{\psi_{1}}^{\frac{1}{2}} C_{\psi_{2}}^{\frac{1}{2}}<\infty .
\end{aligned}
$$

Let $\hat{g}(\omega)=\hat{f}(\omega)\left(C_{\psi_{1}, \psi_{2}}-\int_{R^{d-1}} \int_{A_{1}^{\prime}}^{A_{2}^{\prime}} \overline{\hat{\psi}_{1}\left(A_{a}^{T} S_{s}^{T} \omega\right)} \hat{\psi}_{2}\left(A_{a}^{T} S_{s}^{T} \omega\right) \frac{d a d s}{a^{\frac{d^{2}-d+1}{d}}}\right)$, then we have $g \in L^{2}\left(R^{d}\right)$.

We have

$$
\begin{aligned}
\langle f & \left.-f_{A_{1}^{\prime}, A_{2}^{\prime}}, g\right\rangle \\
& =C_{\psi_{1}, \psi_{2}}^{-1} \int_{R^{d}} \hat{f}(\omega) \overline{\hat{g}(\omega)}\left(C_{\psi_{1}, \psi_{2}}-\int_{R^{d-1}} \int_{A_{1}^{\prime}}^{A_{2}^{\prime}} \overline{\hat{\psi}_{1}\left(A_{a}^{T} S_{s}^{T} \omega\right)} \hat{\psi}_{2}\left(A_{a}^{T} S_{s}^{T} \omega\right) \frac{d a d s}{a^{\frac{d^{2}-d+1}{d}}}\right) d \omega \\
& =C_{\psi_{1}, \psi_{2}}^{-1} \int_{R^{d}}|\hat{f}(\omega)|^{2}\left|C_{\psi_{1}, \psi_{2}}-\int_{R^{d-1}} \int_{A_{1}^{\prime}}^{A_{2}^{\prime}} \overline{\hat{\psi}_{1}\left(A_{a}^{T} S_{s}^{T} \omega\right)} \hat{\psi}_{2}\left(A_{a}^{T} S_{s}^{T} \omega\right) \frac{d a d s}{a^{\frac{d^{2}-d+1}{d}}}\right|^{2} d \omega .
\end{aligned}
$$


But $f(x)=f_{A_{1}^{\prime}, A_{2}^{\prime}}(x)$, hence

$$
C_{\psi_{1}, \psi_{2}}-\int_{R^{d-1}} \int_{A_{1}^{\prime}}^{A_{2}^{\prime}} \overline{\hat{\psi}_{1}\left(A_{a}^{T} S_{s}^{T} \omega\right)} \hat{\psi}_{2}\left(A_{a}^{T} S_{s}^{T} \omega\right) \frac{d a d s}{a^{\frac{d^{2}-d+1}{d}}}=0, \quad \omega \in \operatorname{suup} \hat{f}
$$

Since $\int_{R^{d-1}} \int_{A_{1}^{\prime}}^{A_{2}^{\prime}} \overline{\hat{\psi}_{1}\left(A_{a}^{T} S_{s}^{T} \omega\right)} \hat{\psi}_{2}\left(A_{a}^{T} S_{s}^{T} \omega\right) \frac{d a d s}{a^{\frac{d^{2}-d+1}{d}}}$ is a continuous function on $R^{d}$ with respect to $\omega$, we get

$$
C_{\psi_{1}, \psi_{2}}=\int_{R^{d-1}} \int_{A_{1}^{\prime}}^{A_{2}^{\prime}} \overline{\hat{\psi}_{1}\left(A_{a}^{T} S_{s}^{T} \omega\right)} \hat{\psi}_{2}\left(A_{a}^{T} S_{s}^{T} \omega\right) \frac{d a d s}{a^{\frac{d^{2}-d+1}{d}}}, \quad \omega \in \operatorname{suup} \hat{f}
$$

(iv) $\Rightarrow$ (v):

$$
\begin{aligned}
\mid \sigma & (p, q, r) f(x)-C_{\psi_{1}, \psi_{2}}^{-1} \int_{A_{1}^{\prime} p}^{A_{2}^{\prime} p} d a \int_{R^{d-1}} d s \int_{R^{d}}\left\langle\sigma(p, q, r) f, \sigma(a, s, t) \psi_{1}\left|\sigma(a, s, t) \psi_{2}(x) \frac{d t}{a^{d+1}}\right|\right. \\
& =\left|\sigma(p, q, r) f(x)-\sigma(p, q, r) f_{A_{1}^{\prime}, A_{1}^{\prime}}(x)\right| \\
& =|p|^{-\frac{2 d-1}{2 d}}\left|f(x)-f_{A_{1}^{\prime}, A_{1}^{\prime}}(x)\right| \\
& =0 .
\end{aligned}
$$

(v) $\Rightarrow$ (ii), and (ii) $\Rightarrow$ (i) are obvious.

\section{Conclusions and future work}

The results in this paper set the foundation for the study of a number of questions related to the continuous shearlet transform, including the following:

1. A pointwise convergence result on the inverse shearlet transform in arbitrary dimensions.

2. Every pair of admissible shearlets possess the HAP in the sense of $L^{2}\left(R^{d}\right)$.

3. A sufficient condition for the pointwise HAP to hold, which depends on both shearlets and functions to be reconstructed in arbitrary dimensions.

The study of these issues will be the focus of future investigations. In future work, the obtained results can further be generalized to a continuous Toeplitz shearlet transform, and also can be generalized to a continuous generalized shearlet transform.

\section{Competing interests}

The authors declare that they have no competing interests.

Authors' contributions

YS, WS, and WZ finished this work together. The three authors read and approved the final manuscript.

\section{Author details}

${ }^{1}$ School of Mathematics and Statistics, Central South University, Changsha, 410083, China. ${ }^{2}$ School of Mathematical Sciences, Xin Jiang Normal University, No. 102 Xinyi Road, Urumuqi, 830054, China. ${ }^{3}$ Key Laboratory of Digital Earth Science, Institute of Remote Sensing and Digital Earth, Chinese Academy of Sciences, No. 9 Dengzhuang South Road, Haidian District, Beijing, 100094, China. 


\section{References}

1. Candès, EJ, Donoho, DL: Ridgelets: a key to higher-dimensional intermittency? Philos. Trans. R. Soc. Lond. A 357(1760), 2495-2509 (1999)

2. Candès, EJ, Donoho, DL: Curvelets: A Surprisingly Effective Nonadaptive Representation for Objects with Edges. DTIC document, Saint Malo (1999)

3. Do, MN, Vetterli, M: The contourlet transform: an efficient directional multiresolution image representation. IEEE Trans. Image Process. 14(12), 2091-2106 (2005)

4. Guo, K, Kutyniok, G, Labate, D: Sparse multidimensional representations using anisotropic dilation and shear operators. In: Chen, G, Lai, MJ (eds.) Wavelets and Splines (Athens, GA, 2005), pp. 189-201. Nashboro Press, Nashville (2006)

5. Kumara, D, Kumarb, S, Singhd, B: Cone-adapted continuous shearlet transform and reconstruction formula J. Nonlinear Sci. Appl. 9(1), 262-269 (2016)

6. Ramanathan, J, Steger, T: Incompleteness of sparse coherent states. Appl. Comput. Harmon. Anal. 2(2), 148-153 (1995)

7. Liu, B, Sun, WC: Homogeneous approximation property for continuous wavelet transforms. J. Approx. Theory 155(2), 111-124 (2008)

8. Sun, WC: Homogeneous approximation property for wavelet frames. Monatshefte Math. 159(3), $289-324$ (2010)

9. Sun, WC: Homogeneous approximation property for wavelet frames with matrix dilations. Math. Nachr. 283(10), 1488-1505 (2010)

10. Zhao, ZJ, Sun, WC: Homogeneous approximation property for wavelet frames with matrix dilations, II. Acta Math. Sin. 29(1), 183-192 (2013)

11. Heil, CE, Kutyniok, G: The homogeneous approximation property for wavelet frames. J. Approx. Theory 147(1), 28-46 (2007)

12. Heil, CE: History and evolution of the density theorem for Gabor frames. J. Fourier Anal. Appl. 13(2)، 113-166 (2007)

13. Kittipoom, P, Kutyniok, G, Lim, WQ: Irregular shearlet frames: geometry and approximation properties. J. Fourier Anal. Appl. 17(4), 604-639 (2011)

14. Kittipoom, P: Irregular shearlet frames. PhD thesis, Justus Liebig Universität Gießen (2009)

15. Dahlke, S, Häuser, S, Steidl, G, Teschke, G: Shearlet coorbit spaces: traces and embeddings in higher dimensions. Monatshefte Math. 169(1), 15-32 (2013)

16. Su, Y: Heisenberg type uncertainty principle for continuous shearlet transform. J. Nonlinear Sci. Appl. 9(3), 778-786 (2016)

17. Liu, B, Sun, WC: Homogeneous approximation property for multivariate continuous wavelet transforms. Numer Funct. Anal. Optim. 30(7-8), 784-798 (2009)

18. Dahlke, S, Kutyniok, G, Maass, P, Sagiv, C, Stark, HG, Teschke, G: The uncertainty principle associated with the continuous shearlet transform. Int. J. Wavelets Multiresolut. Inf. Process. 6(2) 157-181 (2008)

\section{Submit your manuscript to a SpringerOpen ${ }^{\circ}$ journal and benefit from:}

- Convenient online submission

- Rigorous peer review

- Immediate publication on acceptance

- Open access: articles freely available online

High visibility within the field

Retaining the copyright to your article 